\title{
CINÉTICA DA DISSOLUÇÃO DE PRECIPITADOS DURANTE O REAQUECIMENTO DE PLACAS DE AÇO MICROLIGADO
}

Antonio Augusto Gorni

\section{Resumo}

Desenvolveu-se um modelo matemático para determinar a cinética de solubilização de elementos microligantes durante o reaquecimento de placas de aço para a laminação a quente. $O$ modelo permite verificar que, sob condições práticas de reaquecimento de placas, precipitados eutéticos com tamanho superior a $250 \mathrm{~nm}$ não serão completamente dissolvidos, reduzindo o teor de Nb solubilizado na austenita. Também verifica-se que a eficiência da solubilização varia em função de pequenas oscilações na composição química de aços microligados, fato que é particularmente importante nas chamadas "marcas de skid" (regiões mais frias) das placas reaquecidas.

Palavras-chave: Solubilização; Cinética; Aço microligado; Modelo matemático.

\section{DISSOLUTION KINETICS OF PRECIPITATES DURING MICROALLOYED STEEL SLAB REHEATING}

\begin{abstract}
This work describes a mathematical model for the calculation of the solution kinetics of microalloying elements during steel slab reheating previous to hot rolling. That model shows that, under the industrial practical conditions of slab reheating, eutectoid precipitates with size greater than $250 \mathrm{~nm}$ are not completely dissolved, decreasing the free $\mathrm{Nb}$ content in austenite. It is also verified that the solution efficiency varies according to small oscillations in chemical composition of the microalloyed steels, a situation that is particularly important in the so called "skid marks" (colder regions) in the reheated slabs.
\end{abstract}

Keywords: Solution; Kinetics; Microalloyed steel; Mathematical model.

\section{INTRODUÇÃO}

A solubilização plena do $\mathrm{Nb}$ e de outros elementos microligantes durante a austenitização é uma condição essencial para que os aços estruturais ARBL (Alta Resistência e Baixa Liga) respondam adequadamente à laminação controlada e desenvolvam as propriedades mecânicas esperadas. Ao longo das últimas décadas foram desenvolvidos inúmeros estudos sobre a solubilidade dos elementos microligantes, particularmente sobre as equações que permitem calcular os teores de equilíbrio dos microligantes solubilizados na austenita em função da temperatura. ${ }^{(1)}$

Por outro lado, poucos estudos foram feitos sobre a cinética de dissolução dos precipitados de elementos microligantes nos aços, muito provavelmente devido às grandes dificuldades experimentais envolvidas em sua análise metalográfica. $\mathrm{Na}$ prática considera-se, geralmente, que são necessários de 10 minutos a 30 minutos sob a temperatura mínima prevista pelas equações termodinâmicas de solubilidade para que ocorra a solução completa dos precipitados na austenita. Contudo, é muito conveniente ter uma idéia mais precisa do tempo mínimo necessário para a solubilização. Neste caso, pode-se minimizar o tempo de reaquecimento dos aços microligados, reduzindo seu custo de produção e aumentando a produtividade da linha.

A microestrutura das placas de aços ARBL produzidas por lingotamento contínuo apresenta precipitados de elementos microligantes com vários tamanhos, formatos e composições químicas ao longo da espessura do semi-produto. ${ }^{(2,3)}$ No caso específico de um aço microligado ao $\mathrm{NbTiV}^{(2)}$ foi verificado que $80 \%$ dos precipitados são esféricos e medem entre $1 \mathrm{~nm}$ e $150 \mathrm{~nm}$; $10 \%$ são cúbicos, medindo entre $60 \mathrm{~nm}$ e $300 \mathrm{~nm}$; e os restantes $10 \%$ apresentam formato de estrela ou asa, apresentando tamanhos entre $150 \mathrm{~nm}$ e $300 \mathrm{~nm}$. A região interdendrítica apresenta maior concentração de precipitados, uma vez

Membro da ABM. Engenheiro de Materiais, M. Eng., Dr. Eng., Especialista da Gerência de Suporte Técnico da Laminação a Quente, Usiminas-Cubatão, Rodovia Cônego Domênico Rangoni s/n, Bairro Jardim das Indústrias, Cep II573-900, Cubatão, SP, Brasil. E-mail: antonio.gorni@usiminas.com 
que o $\mathrm{Nb}$ apresenta forte tendência à segregação devido a seu alto índice de partição: o aço líquido interdendrítico apresenta teor desse elemento sete vezes superior à composição química nominal da liga. Essa distribuição irregular de precipitados provoca variações locais na eficácia dos precipitados em conter o crescimento de grão (o chamado efeito de Zener pinning) e na velocidade de sua dissolução durante o reaquecimento da placa.

De fato, o tempo necessário para a dissolução de precipitados está diretamente associado ao seu tamanho. Uma análise microestrutural de bobinas a quente de aços microligados nacionais ${ }^{(4)}$ revelou a presença de precipitados eutéticos grosseiros com tamanho superior a $500 \mathrm{~nm}$. A fração e composição química desses precipitados permitiram estimar que apenas de $51 \%$ a $67 \%$ dos elementos microligantes foram solubilizados, restando portanto de $33 \%$ a $49 \%$ na forma combinada, inertes do ponto de vista metalúrgico. Obviamente, isso constitui um significativo desperdício.

Essa situação motivou o desenvolvimento de um modelo matemático para calcular a cinética de solubilização dos elementos microligantes sob as condições típicas de reaquecimento das placas de aços estruturais, com o objetivo de se caracterizar melhor os possíveis efeitos decorrentes de variações do tamanho de precipitados, da composição química e da temperatura na solubilização desses elementos.

\section{DESENVOLVIMENTO DO MODELO DE CINÉTICA DE SOLUBILIZAÇÃO}

O modelo matemático aqui desenvolvido baseia-se na proposta de Andersen e Grong, ${ }^{(5)}$ onde são consideradas as seguintes condições: precipitados esféricos, fluxo de solução controlado pela difusão do átomo mais lento e precipitados suficientemente distantes de forma que não houvesse interação entre seus fluxos de difusão. É adotada a formulação de cinética de dissolução não-isotérmica, que é a situação representativa para o reaquecimento de placas. Neste caso, a evolução do raio $r$ do precipitado ao longo do tempo é dada pela fórmula

$$
r^{2}=r_{0}^{2}-2 \int_{t_{1}}^{t_{2}} \alpha D_{m} d t
$$

onde $r_{0}$ é o raio inicial do precipitado, $D_{m}$ é a difusividade do elemento que está sendo solubilizado e $\alpha$ é um fator que define $\circ$ potencial termodinâmico para $\circ$ processo através da supersaturação do elemento que está sendo solubilizado, sendo:

$$
\propto=\frac{C_{i}-C_{m}}{C_{0}-C_{i}}
$$

onde $C_{i}$ é a concentração de equilíbrio do soluto na interface precipitado/matriz, $C_{m}$ é a concentração de soluto na matriz e $C_{0}$ é a composição química nominal da liga. Já a evolução da fração $f$ dos precipitados ao longo do tempo é dada pela fórmula:

$$
f=f_{0}\left(I-\frac{2}{r_{0}^{2}} \int_{t_{1}}^{t_{2}} \alpha D_{m} d t\right)^{3 / 2}
$$

onde $f_{0}$ é a fração inicial de precipitados presente na microestrutura.

A dissolução não-isotérmica implica numa complicação adicional, já que a fração de precipitados e sua composição química (e, consequentemente, da matriz) são funções da temperatura. Neste caso, é necessário empregar um modelo matemático para calcular o equilíbrio termodinâmico entre os precipitados de elementos microligantes e a austenita, que já havia sido desenvolvido em outra oportunidade. ${ }^{(6)}$

Essa formulação matemática foi implementada num programa em Visual Basic, permitindo interação amigável com o usuário, inclusive com a geração de gráficos.

\section{APLICAÇÕES DO MODELO DE CINÉTICA DE SOLUBILIZAÇÃO}

Os relativamente poucos estudos sobre a cinética da dissolução de precipitados geralmente mantêm seu foco na microestrutura, ou seja, eles analisam a evolução do diâmetro e da fração dos precipitados ao longo do tempo. As aplicações descritas neste trabalho expressam a dissolução através do teor de Nb solubilizado na austenita, um parâmetro muito mais significativo do ponto de vista industrial.

A curva de aquecimento adotada nos cálculos é típica de condições industriais, tendo sido considerada aqui a pior situação em termos de encharque térmico. Ou seja: foi considerada a evolução da temperatura na metade da espessura da placa e no ponto de seu comprimento onde ela se encontra apoiada sobre o skid do forno de reaquecimento. Esse skid é refrigerado a água, roubando calor da placa nesse ponto específico e reduzindo localmente sua temperatura.

\section{I Efeito do Tamanho do Precipitado}

Para esta análise foi considerado um aço microligado hipotético, comercialmente representativo, que apresenta a seguinte composição química: $0,11 \% \mathrm{C}$, $0,050 \% \mathrm{Nb}, 0,016 \% \mathrm{Tie} 0,0043 \% \mathrm{~N}$. Foram considerados três tamanhos de precipitado: médio ( $17 \mathrm{~nm}$, conforme Borggren et al. ${ }^{(3)}$ para aço ao NbTi) e eutéticos (250 e $500 \mathrm{~nm})^{(2-4)}$ 
Conforme mostrado na Figura I, os precipitados de tamanho médio apresentam dissolução do $\mathrm{Nb}$ na austenita quase que imediata a partir de aproximadamente $975^{\circ} \mathrm{C}$ para a pior condição de reaquecimento industrial da placa. Já os precipitados eutéticos com $250 \mathrm{~nm}$ apresentam cinética bem mais lenta, havendo uma defasagem entre os teores solubilizados previstos pelo modelo termodinâmico e pelo modelo cinético durante quase todo o tempo de reaquecimento, exceto em seus momentos finais, quando finalmente ocorre a solubilização plena do $\mathrm{Nb}$. Já no caso dos precipitados eutéticos de $500 \mathrm{~nm}$ o modelo de cinética de solubilização prevê que não haverá solubilização total desse elemento ao final do reaquecimento
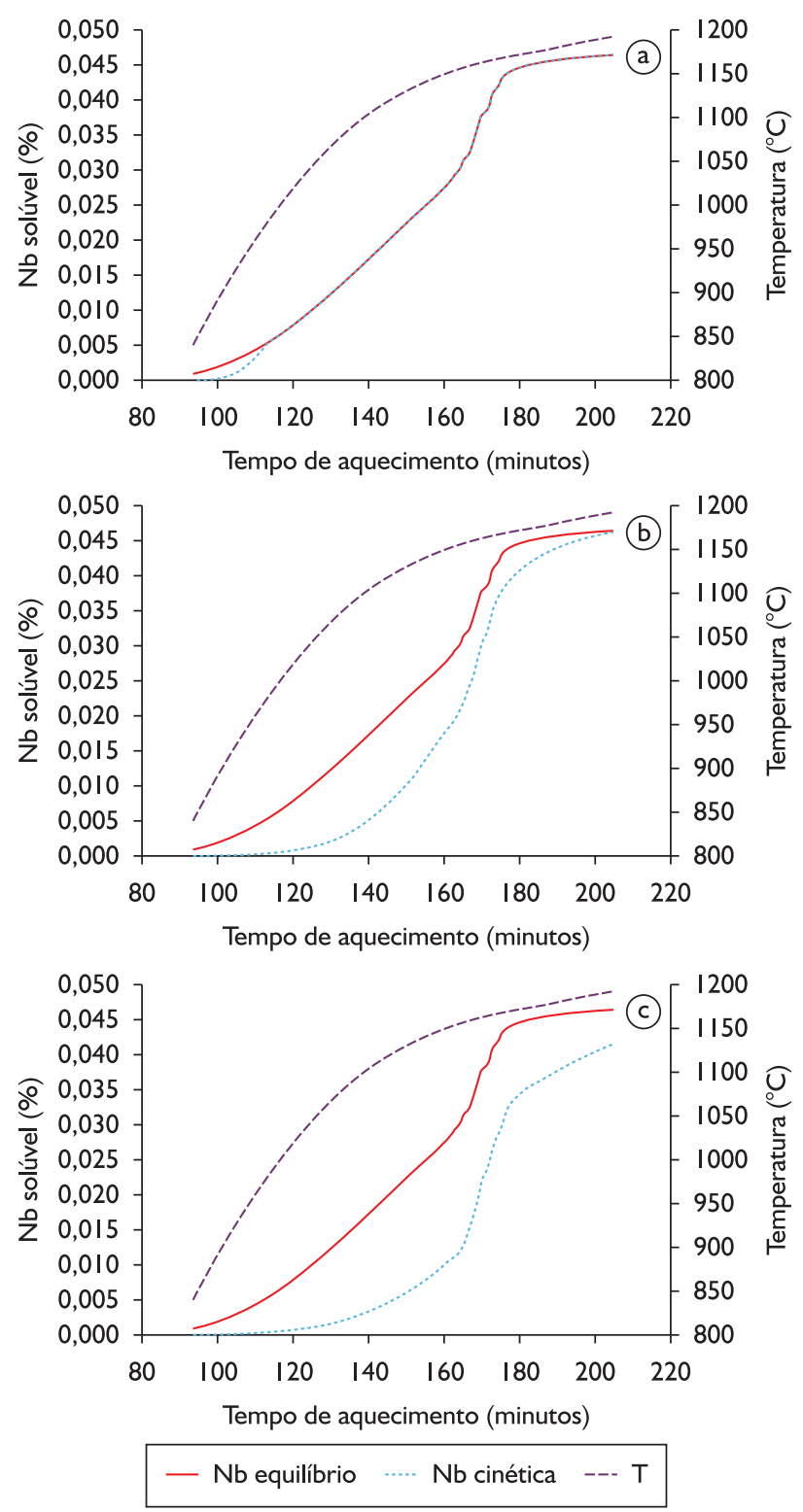

Figura I. Evolução da solubilização prevista do $\mathrm{Nb}$ e da temperatura no interior da placa ao longo do tempo de reaquecimento para o aço aqui estudado. Tamanho de precipitado: a) $17 \mathrm{~nm}$; b) $250 \mathrm{~nm}$; e c) $500 \mathrm{~nm}$. da placa. Portanto, para esta liga específica, as condições de solidificação durante o lingotamento contínuo devem, tanto quanto possível, evitar a formação de precipitados eutéticos com tamanho superior a $250 \mathrm{~nm}$.

Estes resultados são confirmados por modelos industriais usados para prever as propriedades mecânicas de laminados planos fabricados com aços microligados, tais como o Structura (IRSID) $)^{(7)}$ e Tacsi (ArcelorMittal). (8) Eles desconsideram a cinética de solubilização dos elementos microligantes durante $\circ$ reaquecimento da placa, calculando o teor solubilizado de elementos microligantes a partir do modelo termodinâmico de equilíbrio, exclusivamente em função da composição química do aço e da temperatura por ele alcançada.

\subsection{Efeito da Composição da Liga}

A seguir é estudado o efeito que pequenas variações na composição química dos aços microligados poderiam exercer na solubilização do Nb na austenita. São propostos casos relevantes de flutuação de composição química, tomando-se como base $o$ aço estudado no item anterior, que estão mostrados na Tabela I. A liga Média corresponde ao aço já citado. As ligas Mínima e Máxima correspondem às composições químicas com os teores de todos os elementos no valor mínimo ou máximo, respectivamente, dentro da faixa aqui estudada. A liga ProMicr possui teores mínimos de elementos intersticiais e teores máximos de elementos microligantes, enquanto que, na liga Prolnt, ocorre exatamente o contrário.

Tabela I. Ligas consideradas para o estudo do efeito de flutuações na composição química sobre a solubilização do Nb em aços microligados. Teores expressos em percentagem em peso

\begin{tabular}{ccccc}
\hline Liga & $\mathbf{C}$ & $\mathbf{N b}$ & $\mathbf{T i}$ & $\mathbf{N}$ \\
\hline Média & 0,11 & 0,050 & 0,016 & 0,0043 \\
Mínima & 0,09 & 0,045 & 0,010 & 0,0005 \\
Máxima & 0,12 & 0,055 & 0,022 & 0,0080 \\
ProMicr & 0,09 & 0,055 & 0,022 & 0,0005 \\
Prolnt & 0,12 & 0,045 & 0,010 & 0,0080 \\
\hline
\end{tabular}

A Figura 2 mostra a evolução do teor de Nb solúvel e da taxa de sua solubilização ao longo da temperatura para as ligas apresentadas na Tabela I. Como se pode observar, a evolução do teor desse elemento microligante ao longo da temperatura apresenta variações conforme a composição química específica do aço. A liga Prolnt apresenta a pior evolução de solubilidade ao longo da temperatura: lenta e limitada, apresentando teor mínimo de Nb solúvel $(0,042 \%)$ a $1.250^{\circ} \mathrm{C}$. A liga Média é a mais conveniente e confiável do ponto de vista industrial, tendo apresentado rápida solubilização em faixa de temperaturas relativamente baixas $\left(1.150^{\circ} \mathrm{C}-1.170^{\circ} \mathrm{C}\right)$, seguida de leve crescimento no teor de $\mathrm{Nb}$ solubilizado para maiores temperaturas. 

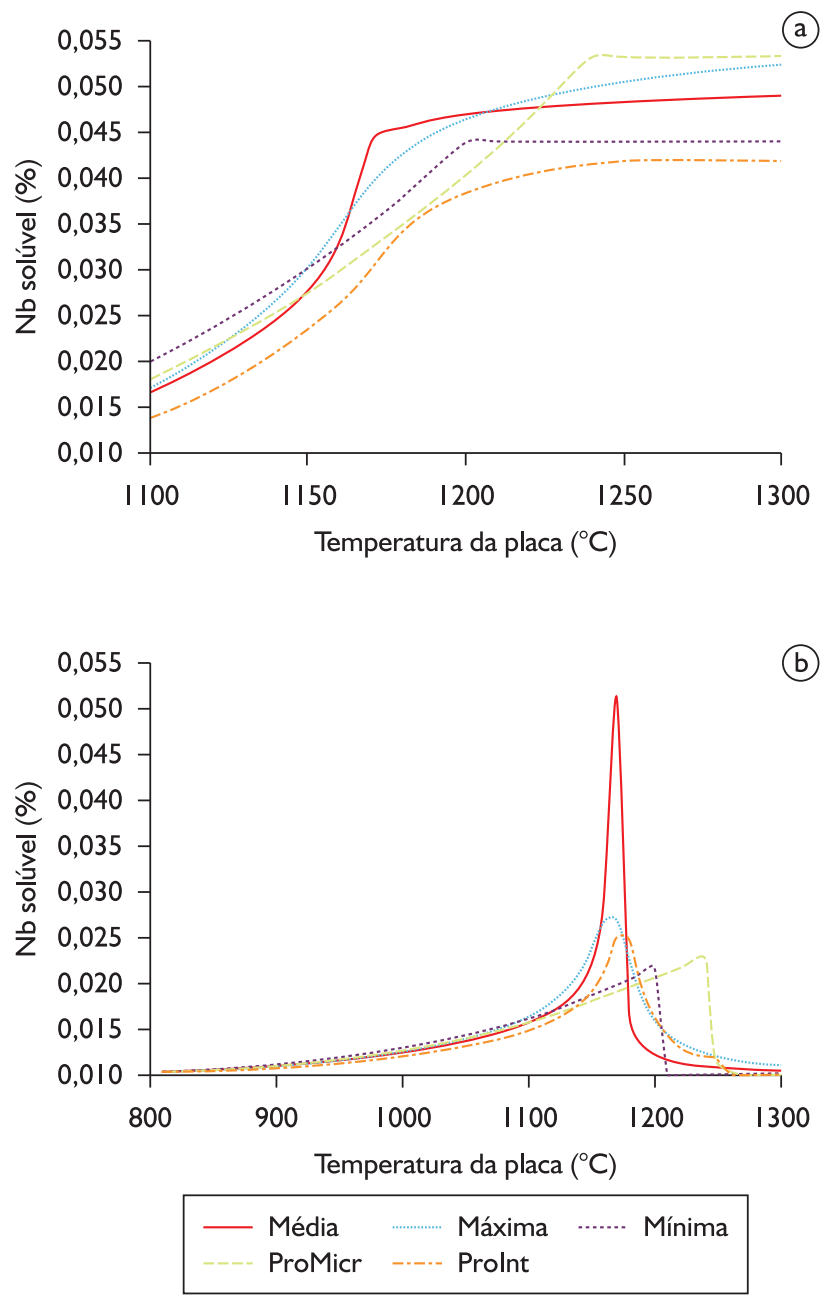

Figura 2. a) Evolução do teor de Nb solubilizado ao longo da temperatura e b) taxa de sua solubilização em relação à temperatura para as ligas mostradas na Tabela I.

Já as ligas Máxima e ProMicr exigiram maiores temperaturas para atingir os mesmos níveis de solubilização relativos à liga Média, mas permitem a obtenção de maiores teores de $\mathrm{Nb}$ solúvel em relação a ela, desde que a temperatura de reaquecimento seja superior a I.210 $0^{\circ} \mathrm{C}$, já que o teor de $\mathrm{Nb}$ total é maior para as duas primeiras ligas. A liga Mínima apresenta solubilização mais rápida do $\mathrm{Nb}$ até $1.150^{\circ} \mathrm{C}$, mas é ultrapassada a partir dessa temperatura por todas as demais, exceto a Prolnt.

Como se pode observar, variações relativamente pequenas nos teores dos elementos envolvidos nos precipitados de elementos microligantes $(\mathrm{Nb}, \mathrm{Ti}, \mathrm{C} \mathrm{e} \mathrm{N})$ podem conduzir a alterações significativas na evolução da solubilização do $\mathrm{Nb}$ ao longo do reaquecimento da placa, o que se pode refletir na resposta do aço à laminação controlada e, consequentemente, nas propriedades mecânicas do produto final.

\subsection{Efeito das Marcas de Skid em Função da Composição Química}

Durante o reaquecimento industrial de placas de aço é inevitável que elas apresentem menores temperaturas nas regiões onde se encontram apoiadas nos skids refrigerados a água do forno. As diferenças de temperatura ao longo da placa podem ser significativas: por exemplo, no momento do desenfornamento, o núcleo da placa pode estar a $1.225^{\circ} \mathrm{C}$, enquanto que o mesmo local na região dos skids apresenta temperatura de $1.195^{\circ} \mathrm{C}-$ portanto, uma diferença de $30^{\circ} \mathrm{C}$.

A Tabela 2 mostra os teores calculados de Nb solubilizado no momento do desenfornamento para esses dois pontos da placa (na região do skid e longe dela) considerando as ligas apresentadas na Tabela I. Como já esperado, a diferença no teor de $\mathrm{Nb}$ solubilizado na austenita entre esses dois pontos da placa é função da composição química da liga. A diferença observada é mínima no caso da liga Média e máxima, no caso da liga ProMicr, cujo grau máximo de solubilização só é alcançado para temperaturas muito altas, da ordem de $1.240^{\circ} \mathrm{C}$. A liga Prolnt também apresenta flutuação significativa nos teores de Nb solúvel entre as regiões da placa fora e dentro da região do skid.

Tabela 2. Diferença nos teores de Nb solubilizado no núcleo da placa em função da variação na temperatura de desenfornamento causada pelas marcas de skid e por oscilações na composição química do aço

\begin{tabular}{ccccc}
\hline Liga & \multicolumn{2}{c}{$\mathbf{N b}_{\text {sol }}$} & $\begin{array}{c}\Delta \mathbf{N b}_{\text {sol }} \\
{[\% \text { peso }]}\end{array}$ & $\begin{array}{c}\Delta \mathbf{N b}_{\text {sol }} \\
{[\% \text { peso }]}\end{array}$ \\
\cline { 2 - 3 } & $\mathbf{I . 2 2 5 ^ { \circ } \mathbf { C }}$ & $\mathbf{1 . 1 9 5 ^ { \circ } \mathbf { C }}$ & $-0,002$ & -4 \\
\hline Média & 0,048 & 0,046 & -048 & -5 \\
Mínima & 0,044 & 0,042 & $-0,002$ & -8 \\
Máxima & 0,049 & 0,045 & $-0,004$ & -10 \\
Prolnt & 0,041 & 0,037 & $-0,004$ & -24 \\
ProMicr & 0,050 & 0,038 & $-0,012$ & \\
\hline
\end{tabular}

\subsection{Efeito na Composição Química no Tamanho Máximo de Precipitado Solubilizável}

As variações na solubilidade do $\mathrm{Nb}$ ao longo da temperatura em função da composição química da liga, já apresentadas nos itens anteriores, também acabam alterando o tamanho máximo de precipitado que pode ser dissolvido na austenita durante o reaquecimento da placa. Já foi visto no item 3.I que, no caso da liga Média e considerando as condições de reaquecimento lá descritas, podem ser dissolvidos precipitados eutéticos de até $250 \mathrm{~nm}$. Mais uma vez esta liga mostra um comportamento muito adequado, pois este é o melhor caso dentre todas as ligas mostradas na Tabela I. De acordo com o modelo cinético desenvolvido neste caso, o tamanho máximo de preci- 
pitado que pode ser solubilizado para as condições de reaquecimento industrial consideradas aqui é decrescente conforme a sequência de ligas a seguir: Máxima (225 nm), ProMicr (200 nm), Mínima (I 75 nm) e Prolnt (I 50 nm).

Estes resultados indicam que ligas com teores mais baixos e menos balanceados de $\mathrm{Nb}, \mathrm{Ti}, \mathrm{C}$ e $\mathrm{N}$ tendem a apresentar menor potencial de difusão na austenita, retardando a solubilização de seus precipitados.

\section{CONCLUSÕES}

A análise dos aspectos cinéticos e termodinâmicos da solubilização dos elementos microligantes durante o reaquecimento de placas de aço evidencia que devem ser levados em conta diversos parâmetros para se conseguir um teor consistente de $\mathrm{Nb}$ solúvel no momento do desenfornamento:

- composição química balanceada: ela deve propiciar rápida solubilização do $\mathrm{Nb}$ sob as menores temperaturas possíveis pela maximização do potencial termodinâmico de difusão desse elemento;
- morfologia dos precipitados na microestrutura bruta de fusão da placa: os precipitados eutéticos grosseiros devem ser refinados e sua fração volumétrica minimizada;

- condições de reaquecimento: o processo de reaquecimento industrial deve garantir que 0 núcleo da placa atinja a temperatura mínima para solubilização plena do $\mathrm{Nb}$, inclusive nas regiões das marcas de skid. Além disso, deve ser minimizado o gradiente de temperatura entre essas regiões e o restante da placa.

A otimização da solubilização dos elementos microligantes durante o reaquecimento de placas é uma medida fundamental para garantir maior confiabilidade do processo de laminação controlada, levando à fabricação de produtos com menor dispersão em suas propriedades mecânicas. Este é um ponto vital para o atendimento ao cliente e para a garantia da competitividade das usinas.

\section{REFERÊNCIAS}

I GLADMAN, T. The physical metallurgy of microalloyed steels. London: Ashgate, 1997.

2 CHAKRABARTI, D. et al. Development of bimodal grain structures in nb-containing high-strength low-alloy steels during slab reheating. Metallurgical and Materials Transactions A, v. 39A, n. 8, p. 1963-77, Aug. 2008. http://dx.doi.org/I0.1007/s I I66 I-008-9535-0

3 BORGGREN, U. et al. A model for particle dissolution and precipitation in HSLA steels. Advanced Materials Research, v. I5- I7, p. 7|4-9, 2007. http://dx.doi.org/10.4028/www.scientific.net/AMR. I5-I7.7|4

4 GALLEGO, J. et al. Carbonitretos eutéticos em aços microligados comerciais. In: CONGRESSO BRASILEIRO DE ENGENHARIA E CIÊNCIA DOS MATERIAIS - CBECIMAT, 19., 2002, Natal. Anais... São Paulo: Metallum, 2002. p. 2380-6.

5 ANDERSEN, I.; GRONG, O. Analytical modelling of grain growth in metals and alloys in the presence of growing and dissolving precipitates - I. Normal grain growth. Acta Metallurgica et Materialia, v. 43, n. 7, p. 2673-88, July 1995. http://dx.doi.org/I0.1016/0956-7I5I(94)00488-4

6 GORNI, A. A. Spreadsheet applications in materials science. In: FILBY, G. (Ed.) Spreadsheets in science and engineering. Berlin: Springer, 1997. p. 229-60.

7 PIETTE, M.; PERDRIX, C. An integrated model for microstructural evolution in the hot strip mill and tensile properties prediction of plain and microalloyed C-Mn Hot Strip. Materials Science Forum, v. 284-286, p. 36I-8, I998. http://dx.doi.org/I0.4028/www.scientific.net/MSF.284-286.36 I

8 HUIN, D. et al. Microstructural modelling for NbTi micro-alloying adjustement on hot rolled HSLA steels. In: INTERNATIONAL CONFERENCE ON THERMOMECHANICAL PROCESSING OF STEELS - TMP 2008, Padua. Proceedings. Padua: Associazione Italiana di Metallurgia, 2008.

Recebido em: 10/0I/201।

Aprovado em: 16/3/201 I 